\title{
Cantilever Beam Metastructure for Passive Broadband Vibration Suppression
}

\author{
Ratiba Fatma Ghachi \\ rg1408372@qu.edu.qa \\ Department of Civil and Architectural Engineering, Qatar University, Doha, Qatar
}

Wael Alnahhal

wael.alnahhal@qu.edu.qa

Department of Civil and Architectural Engineering, Qatar University, Doha, Qatar

Osama Abdeljaber

osama.abdeljaber@lnu.se

Department of Building Technology, Faculty of Technology, Linnaeus University, Vaxjo, Sweden

\begin{abstract}
This paper presents a beam structure of a new metamaterial-inspired dynamic vibration attenuation system. The proposed experimental research presents a designed cantilevered zigzag structure that can have natural frequency orders of magnitude lower than a simple cantilever of the same scale. The proposed vibration attenuation system relies on the masses placed on the zigzag structure thus changing the dynamic response of the system. The zigzag plates are integrated into the host structure namely a cantilever beam with openings, forming what is referred to here as a metastructure. Experimental frequency response function results are shown comparing the response of the structure to depending on the natural frequency of the zigzag structures. Results show that the distributed inserts in the system can split the peak response of the structure into two separate peaks rendering the peak frequency a low transmission frequency. These preliminary results provide a view of the potential of research work on active-controlled structures and nonlinear insert-structure interaction for vibration attenuation.
\end{abstract}

Keywords: Vibration attenuation; Metastructures; Cantilever beam; Frequency-response-function

\section{INTRODUCTION}

For decades a special interest has been given to vibration control (F. Casadei, Beck, Cunefare, \& Ruzzene, 2012), noise reduction (Filippo Casadei, Dozio, Ruzzene, \& Cunefare, 2010), and wave guiding (Vasseur et al., 2007). Passive vibration suppression is when a system is designed to operate independently from external force or controller. An example of a single DF vibration absorber is if the desired system is modeled as a single degree of freedom oscillator. With the estimation of the stiffness and mass matrix, a single DOF absorber is chosen with stiffness and mass matrix that allows the resonant frequency to be reduced or eliminated (Abdeljaber, Avci, Kiranyaz \& Inman, 2017; "Engineering vibration," 1994). Later, researchers expanded the concept of the single absorber to a series of distributed absorbers distributed along with the structure. These structures are mentioned here as metastructures as their behavior is reminiscent of this of matematerials. The purpose of this research is to reduce the vibration in the structure while allowing it to perform to its original function. 
Zhu et al. (2014) proposed a metastructure design using chiral oscillators (Zhu, Liu, Hu, Sun, \& Huang, 2014). Sun et al. (2010) conducted a numerical and analytical study of beam metastuctures (Sun, Du, \& Pai, 2010). In applied mechanics research, Chen et al. (2011) performed experiments on sandwiched beams damped with spring-mass resonators (Chen, Sharma \& Sun, 2011).

In this paper, we will report experiments on a cantilever beam including zigzags and present the gain in vibration attenuation compared to a cantilever beam of the same scale. The masses placed at the end of the zigzag approach the zigzag's natural frequency to this of the beam, which will change the overall system response.

\section{EXPERIMENTAL ANALYSIS}

The zigzag plates are integrated into the host structure namely a cantilever beam with openings, forming what is referred to here as a metastructure. Experiments were performed on a cantilever beam with zigzags and beams with zigzags and end masses (Figure 1).

Table 1: Geometry and mechanical properties for the host structure

\begin{tabular}{lllll|}
\hline Material properties & Symbol & Value & Units \\
\hline Young's Modulus & $\boldsymbol{E}$ & 69 & $\mathrm{GPa}$ \\
Shear modulus & $\boldsymbol{G}$ & 26 & $\mathrm{GPa}$ & $\mathrm{g} / \mathrm{cm}^{3}$ \\
Density & $\rho$ & 2.7 & $\mathrm{~cm}$ \\
\hline Channel Dimensions & & & $\mathrm{cm}$ \\
\hline Length & $\boldsymbol{L}$ & 80 & $\mathrm{~cm}$ \\
Base Width & $\boldsymbol{o}_{\mathbf{1}}$ & 5 & $\mathrm{~mm}$ \\
Leg Length & $\boldsymbol{O}_{\mathbf{3}}$ & 2.6 & \\
Thickness & $\boldsymbol{o}_{\mathbf{4}}$ & 3.75 & $\mathrm{~cm}$ \\
\hline Cutout Dimensions & & & $\mathrm{cm}$ \\
Length & $\boldsymbol{O}_{\mathbf{5}}$ & 7.1 & - \\
Width & $\boldsymbol{O}_{\mathbf{6}}$ & 3.4 & \\
Number of cut-outs & $\mathbf{N}_{\boldsymbol{c}}$ & 10 & \\
\hline
\end{tabular}

Table 2: Geometry for the zigzag inserts

\begin{tabular}{|llll|}
\hline Zigzag Parameters & Symbol & Value & Units \\
\hline a-beam Length & $\boldsymbol{a}$ & 5.21 & $\mathrm{~mm}$ \\
a-beam Width & $\boldsymbol{\omega}_{\boldsymbol{a}}$ & 1.24 & $\mathrm{~mm}$ \\
b-beam Length & $\boldsymbol{b}$ & 1.73 & $\mathrm{~mm}$ \\
b-beam Width & $\boldsymbol{\omega}_{\boldsymbol{b}}$ & 0.89 & $\mathrm{~mm}$ \\
Thickness & $\boldsymbol{h}$ & 1.9 & $\mathrm{~mm}$ \\
Number of a-beams & $\boldsymbol{N}_{\boldsymbol{a}}$ & 7 & - \\
\hline
\end{tabular}




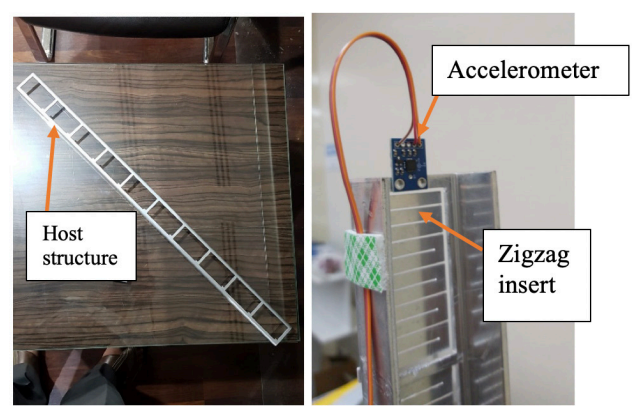

Figure 1: Experimental setup

All components of the cantilever beam were fabricated from 1100-H24 (ASTM, 2019). The host structure consists of a u-channel with openings cut with CNC highpressure waterjet. The waterjet was also used to cut the zigzags out of a $1.9 \mathrm{~mm}$ plate. A Cyanoacrylate has been used to glue the inserts to the frame. Beam and insert geometry are specified in Tables 1 and 2.

\section{RESULTS}

The beam has been subjected to a sweep vibration at its base 1-50 Hz. The input and output signals have been measured using accelerometers. The shaker vibration is perpendicular to the zigzag surface. The shaker is controlled by an amplifier. Given the two signals, a frequency response has been computed and illustrated in Figure 2. The solid line represents the host structure with empty inserts. The dotted line represents the host structure with inserts with an end mass of eight grams. 2315

$$
F R F(\omega)=\frac{\left|A_{\text {out }}(\omega)\right|}{\left|F_{\text {in }}(\omega)\right|}
$$

In the no mass FRF there is one peak at $23 \mathrm{~Hz}$. Compared to a split in peaks in the with mass FRF we can clearly see two peaks one at $15 \mathrm{~Hz}$ and another at $23 \mathrm{~Hz}$.

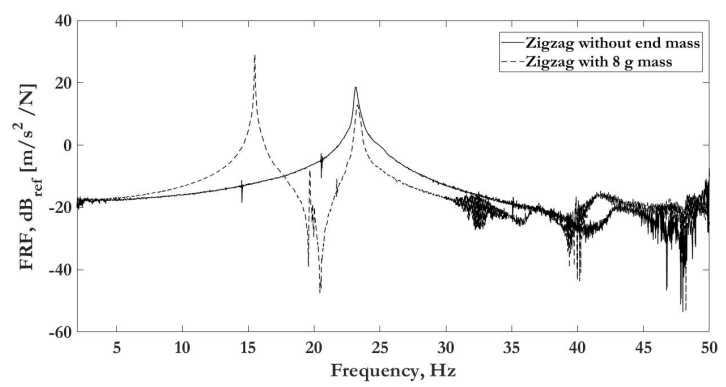

Figure 2: Comparative frequency response function

The phenomenon of the split of the frequency in the no mass FRF is that there is one peak at $23 \mathrm{~Hz}$. Compared to a split in peaks in the with mass FRF, we can clearly see two peaks one at $15 \mathrm{~Hz}$ and another at $23 \mathrm{~Hz}$. The phenomenon that occurs here is that the closer the mass and stiffness of the insert approach the structure own mass and stiffness, the more change we can see in the frequency response peak. This can be achieved by 
controlling the end mass weight for instance. The potential of this observation is that the stiffness and mass of the insert can be optimized to produce a low-frequency zone on the exact frequency the beam normally resonates.

\section{CONCLUSION}

In this paper, we present an experimental investigation on the transmission behavior of a cantilever beam with inserts. Ten inserts have been placed on a $0.8 \mathrm{~m}$ beam with openings. Transmission behavior has been measured by accelerometers under the external force of a shaker vibrating in a sweep signal. Experimental frequency response function results are shown comparing the response of the beam to depending on the natural frequency of the zigzag structures. Results show that the host with inserts system can split the peak response of the structure into two separate peaks rendering the peak frequency a low transmission frequency. These results show the promising potential of research endeavors on active-controlled structures and nonlinear insert-structure interaction for vibration attenuation.

\section{REFERENCES}

Abdeljaber, O., Avci, O., Kiranyaz, S. \& Inman, D. J. (2017). Optimization of linear zigzag insert metastructures for low-frequency vibration attenuation using genetic algorithms. Mechanical Systems and Signal Processing, 84, 625-641. https://doi.org/10.1016/j.ymssp.2016.07.011.

$\operatorname{ASTM}(2019)$.ASTMB209-14: Standard specification for aluminum and aluminum-alloy sheet and plate. ASTM International, 8(2), 2019. https://doi.org/10.22201/fq.18708404e.2004.3.66178.

Casadei, F., Beck, B. S., Cunefare, K. a. \& Ruzzene, M. (2012). Vibration control of plates through hybrid configurations of periodic piezoelectric shunts. Journal of Intelligent Material Systems and Structures, 23(10), 1169-1177. https://doi.org/10.1177/1045389X12443014.

Casadei, F., Dozio, L., Ruzzene, M. \& Cunefare, K. A. (2010). Periodic shunted arrays for the control of noise radiation in an enclosure. Journal of Sound and Vibration, 329(18), 36323646. https://doi.org/10.1016/j.jsv.2010.04.003.

Chen, J. S., Sharma, B. \& Sun, C. T. (2011). Dynamic behaviour of sandwich structure containing spring-mass resonators. Composite Structures, 93(8), 2120-2125. https://doi.org/10.1016/j. compstruct.2011.02.007.

Engineering vibration (1994). Choice Reviews Online, 31(08), 31-4381-31-4381. https://doi. org/10.5860/choice.31-4381.

Sun, H., Du, X. \& Pai, P. F. (2010). Theory of metamaterial beams for broadband vibration absorption. Journal of Intelligent Material Systems and Structures, 21(11), 1085-1101. https:// doi.org/10.1177/1045389X10375637.

Vasseur, J. O., Hladky-Hennion, A. C., Djafari-Rouhani, B., Duval, F., Dubus, B., Pennec, Y. \& Deymier, P. A. (2007). Waveguiding in two-dimensional piezoelectric phononic crystal plates. Journal of Applied Physics, 101(11). https://doi.org/10.1063/1.2740352.

Zhu, R., Liu, X. N., Hu, G. K., Sun, C. T. \& Huang, G. L. (2014). A chiral elastic metamaterial beam for broadband vibration suppression. Journal of Sound and Vibration, 333(10), 27592773. https://doi.org/10.1016/j.jsv.2014.01.009. 